\title{
A GAP THEOREM FOR ABELIAN VARIETIES OVER DIFFERENTIAL FIELDS
}

\author{
Alexandru Buium And Anand Pillay
}

In [B1] the first author proved a theorem about differential algebraic groups (in the sense of Cassidy-Kolchin $[\mathrm{C}],[\mathrm{K} 2]$ ) that implied the geometric LangMordell conjecture [L]; cf. Theorem 1 below. The proof in [B1] used an analytic argument, based on "Big Picard". Hrushovski $[\mathrm{H}]$ was able to replace this analytic argument by a remarkable model theoretic argument, based in its turn on the difficult theory developed in [HZ]. Now Hrushovski's model theoretic methods further lead to striking new results on differential algebraic groups [HS]; cf. Theorem 3 below. So a natural challenge presents itself: to find a proof for these new results that is free from model theory. In this note we shall give in particular a quick analytic proof of Theorem 3, based, again, on "Big Picard". The main result of this note is the "Gap" Theorem 2 below, which should be viewed as a significant complement to Theorem 1; Theorems 1 and 2 will then easily imply Theorem 3 .

Some comments are in order concerning the role of the authors in this paper. The second author asked whether analytic methods as in [B1] could yield strong minimality and local modularity of the "Manin kernel" of a simple abelian variety which does not descend to the constants (Theorem 3 below). The first author then proved strong minimality (assertion 1 of Theorem 3) by proving a weaker version of Theorem 2 (namely for simple abelian varieties). The second author suggested some general ideas, analogous to those in [P1], for obtaining local modularity. In trying to carry out details, the first author came up with the proof of the present Theorem 2, from which everything follows.

Recall some basic terminology of differential algebra [K1], [C], [B2]. (The definitions below will suffice to understand the statements of the Theorems below, without assuming any previous knowledge of differential algebra; for the proofs, however, familiarity with [B1], [B2] is required.) Let $\mathcal{F}$ be a $\delta$-field (i.e. a field of characteristic zero equipped with a derivation $\delta$.) One defines the ring of $\delta$-polynomials $\mathcal{F}\left\{y_{1}, \ldots, y_{N}\right\}$ as the ring of usual polynomials with $\mathcal{F}$-coefficients in the variables $\delta^{i} y_{j}, 1 \leq j \leq N, i \geq 0$. There is an obvious notion of order for $\delta$-polynomials. $\mathcal{F}$ is said to be $\delta$-closed if for any $A, B \in \mathcal{F}\{y\}$, $B \neq 0$, such that the order of $A$ is strictly bigger than the order of $B$ there

\footnotetext{
Received December 2, 1996.

The authors were supported in part by NSF grants DMS-9500331 and DMS-9504788 respectively.
} 
exists $\alpha \in \mathcal{F}$ with $A(\alpha)=0, B(\alpha) \neq 0$. In particular $\mathcal{F}$ and its field of constants $\mathcal{C}$ are algebraically closed. By a $\delta$-closed set of $\mathcal{F}^{N}$ one understands the set of common zeros of a family of $\delta$-polynomials in $\mathcal{F}\left\{y_{1}, \ldots, y_{N}\right\} ; \delta$-closed sets are the closed sets of a Noetherian topology on $\mathcal{F}^{N}$ called the $\delta$-topology (or the Kolchin topology.) We have an induced $\delta$-topology on any affine closed subvariety $X \subset \mathcal{F}^{N}$. More generally if $X$ is any algebraic variety over $\mathcal{F}$ (in the usual sense of algebraic geometry) identify $X$ with its set of $\mathcal{F}$-points and call a subset of $X \delta$-closed if its intersection with the affine Zariski open subsets of $X$ are $\delta$-closed; $\delta$-closed sets of $X$ are, again, the closed sets of a Noetherian topology, the $\delta$-topology. A $\delta$-closed subgroup of an algebraic group is called a differential algebraic group; this definition is equivalent to Kolchin's [K2] due to [P2]. (As a remarkable example, if $A$ is an abelian variety over $\mathcal{F}$, the $\delta$-closure $A^{\sharp}$ of its torsion group is an extremely interesting differential algebraic group that was investigated in detail in [B2]; it is the "incarnation", in this geometry, of the kernel of the Manin map [Man]. Note however that Manin [Man] does not leave the realm of function fields with derivations, which are too small for allowing to even speak about $A^{\sharp}$ as a geometric object; to do so one needs the $\delta$-closed field $\mathcal{F}$. Cf. [B2].) Now for any $\delta$-closed subset $\Sigma$ of an $\mathcal{F}$-variety $X$ one defines the absolute dimension $a(\Sigma) \in N \cup\{\infty\}$ as follows. If $X=A^{N}$, $a(\Sigma)$ is the maximum of the transcendence degrees over $\mathcal{F}$ of the quotient fields of the rings $\mathcal{F}\left\{y_{1}, \ldots, y_{N}\right\} / I\left(\Sigma_{i}\right)$, where $\Sigma_{i}$ are the irreducible components of $\Sigma$ and $I\left(\Sigma_{i}\right)$ is the ideal of all $\delta$-polynomials vanishing on $\Sigma_{i}$; if $X$ is arbitrary one lets $a(\Sigma)$ be the maximum of $a(\Sigma \cap U)$ where $U$ is affine Zariski open in $X$. (Here are a few examples. We have $a(\Sigma)=0$ iff $\Sigma$ is finite. On the other hand $a(X)=\infty$ if $\operatorname{dim} X>0$. Also, if $A$ is an abelian variety of dimension $g$ over $\mathcal{F}$ then $g \leq a\left(A^{\sharp}\right) \leq 2 g$; cf. [B2], Theorem 6.1.)

With these definitions at hand, let us start by reformulating the main result in [B1]:

Theorem 1 [B1]. Let $A$ be an abelian variety over $\mathcal{F}$, that has $\mathcal{F} / \mathcal{C}$-trace zero, and let $\Gamma \subset A$ be a $\delta$-closed subgroup of finite absolute dimension. Then the Zariski closure in $A$ of any $\delta$-closed subset of $\Gamma$ is a finite union of translates of abelian subvarieties.

Recall that an abelian variety over $\mathcal{F}$ is said to have $\mathcal{F} / \mathcal{C}$-trace zero if it contains no non zero abelian subvariety that descends to $\mathcal{C}$.

Consideration of $\delta$-closed subsets of $\Gamma$ that are not Zariski dense in $A$ immediately leads to a proof of the geometric Lang-Mordell conjecture, cf. [B1]. On the other hand Theorem 1 above says nothing about $\delta$-closed subsets of $\Gamma$ that are Zariski dense in $A$. These are the concern of the main result of the present paper, which is the "Gap Theorem" below:

Theorem 2. Let $A$ be an abelian variety over $\mathcal{F}$ of dimension $g$, that has $\mathcal{F} / \mathcal{C}$-trace zero, and let $\Gamma \subset A$ be a $\delta$-closed subgroup of finite absolute dimension. Then any $\delta$-closed subset of $\Gamma$, that is Zariski dense in $A$, has absolute dimension $\geq g+1$. 
Here is the remarkable consequence of Hrushovski's theory refered to in the beginning of this note and for which we shall provide a proof based on Theorems 1 and 2 above.

Theorem 3 [HS]. Let $A$ be a simple abelian variety over $\mathcal{F}$ which does not descend to $\mathcal{C}$. Then the following properties hold:

1) Any proper $\delta$-closed subset of $A^{\sharp}$ is finite.

2) Any $\delta$-closed subset of $A^{\sharp} \times A^{\sharp}$ is a finite union of translates of $\delta$-closed subgroups.

3) If $B$ is another simple abelian variety over $\mathcal{F}$ that is not isogenous to $A$ and does not descend to $\mathcal{C}$ then any proper $\delta$-closed subset of $A^{\sharp} \times B^{\sharp}$ is a finite union of points or points times one of the factors.

Remarks. In the language of model theory, assertion 1 above says that $A^{\sharp}$ is "strongly minimal", assertion 2 implies, as pointed out in [P1], that $A^{\sharp}$ is "locally modular", and assertion 3 says that $A^{\sharp}$ and $B^{\sharp}$ are "orthogonal"; cf [MMP] and [P1]. Theorem 3 implies that any strongly minimal group definable in a differentially closed field (of characteristic 0 ) is either locally modular or nonorthogonal to the field of constants, as well as showing that the nonorthogonality classes of locally modular strongly minimal groups are in 1-1 correspondence with the isogeny classes of simple abelian varieties which do not descend to the constants, these results being originally due to Hrushovski and Sokolovic [HS]. However [HS] contains a deeper trichotomy theorem: that every strongly minimal set in a differentially closed field is "trivial", "locally modular" or nonorthogonal to the field of constants. This depends on showing that such strongly minimal sets are Zariski geometries and then referring to [HZ]. [HZ] has two steps, producing a definable group from a nontrivial (Zariski) strongly minimal set, and then producing a definable field from a non locally modular (Zariski) strongly minimal set. The trichotomy theorem can be deduced from the first step of [HZ] together with the results of the present paper. It would be interesting to find a "direct" proof of the first step of [HZ] in the context of differential fields.

In order to prove Theorems 2 and 3 we start by recalling (a few facts about) the dictionary developed in [B1], [B2]. By a $D$-scheme we understand an $\mathcal{F}$-scheme $V$ together with a lifting of $\delta: \mathcal{F} \rightarrow \mathcal{F}$ to a derivation of $\mathcal{O}_{V}$. $D$-schemes form a category in the obvious way (morphisms are assumed to commute with derivations.) Group objects in this category are called $D$-group schemes. $D$-schemes whose underlying scheme are varieties are called $D$-varieties; $D$-group schemes whose underlying group schemes are algebraic groups are called algebraic $D$-groups. Here varieties and algebraic groups are assumed, by definition, irreducible. Recall that given an $\mathcal{F}$-variety $X$ there is a $D$-scheme $X^{\infty}=\operatorname{jet}_{\infty}(X / \mathcal{F}, \delta)$ satisfying the following universality property:

$$
\operatorname{Hom}_{\mathcal{F}-\text { schemes }}\left(V^{!}, X\right) \simeq \operatorname{Hom}_{D \text {-schemes }}\left(V, X^{\infty}\right)
$$

for any $D$-scheme $V$, where $V^{!}$is the underlying scheme of $V$. There is a bijection $\Sigma \mapsto \Sigma^{[\infty]}, H \mapsto H^{\text {sol }}$ between $\delta$-closed subsets $\Sigma$ of $X$ and reduced 
closed $D$-subschemes $H$ of $X^{\infty}$. In case $H$ is a $D$-subvariety of $X^{\infty}$ we have $a\left(H^{\text {sol }}\right)=\operatorname{dim} H$. If $X$ is an algebraic group then $X^{\infty}$ is a $D$-group scheme and, under the above correspondence, irreducible $\delta$-closed subgroups of finite absolute dimension correspond to algebraic $D$-subgroups.

With the above facts at hand we are already able to check that Theorem 3 follows from Theorems 1 and 2 .

To check assertion 1 assume there is a proper infinite $\delta$-closed subset $\Sigma \subset A^{\sharp}$ and seek a contradiction. We may assume $\Sigma$ is minimal with this property; in particular that it is irreducible. By Theorem 2 we have $a(\Sigma) \geq g+1$. We may assume $0 \in \Sigma$. By [B2], Theorem 5.1, $A^{\sharp}$ is contained in any Zariski dense $\delta$-closed subgroup of $A$. Since $A$ is simple this implies that $\Sigma$ is not a subgroup. Hence either $\Sigma \neq-\Sigma$ or there exists $x \in \Sigma$ such that $\Sigma+x \neq \Sigma$. Set $S:=\Sigma^{[\infty]}, S^{\prime}:=(\Sigma+x)^{[\infty]}, G:=\left(A^{\sharp}\right)^{[\infty]}$. So either $S \neq-S$ or $S \neq S^{\prime}$. Now $G$ is a variety of dimension $\leq 2 g$ and $S, S^{\prime},-S$ are closed subvarieties of $G$ of dimension $\geq g+1$. Since both intersections $S \cap(-S)$ and $S \cap S^{\prime}$ are non empty, they both have dimension at least 2 , in particular none of the intersections is finite. Since one of the intersections is strictly contained in $S$ this contradicts the minimality of $\Sigma$, which closes the proof of assertion 1 .

To check assertions 2 and 3 assume that either $B=A$ or $B$ is simple, not descending to $\mathcal{C}$, and non isogenous to $A$ respectively. Let $\Sigma \subset A^{\sharp} \times B^{\sharp}$ is a proper infinite irreducible $\delta$-closed subset containing 0 and we have to prove that $\Sigma$ is a translate of a $\delta$-closed subgroup (in case 2) or $\Sigma$ is zero times one of the factors (in case 3.) Let $S=\Sigma^{[\infty]}, G=\left(A^{\sharp}\right)^{[\infty]}, H=\left(B^{\sharp}\right)^{[\infty]}$. Since, by assertion 1, $G$ and $H$ contain no proper closed $D$-subvariety of positive dimension it follows that either $S$ is zero times one of the factors of $G \times H$ or $S$ is generically finite over both factors. In the first case both assertions 2 and 3 follow. In the second case we may assume of course $\operatorname{dim} A \leq \operatorname{dim} B$ and we get

$$
a(\Sigma)=\operatorname{dim} S=\operatorname{dim} G=a\left(A^{\sharp}\right) \leq 2 \cdot \operatorname{dim} A \leq \operatorname{dim} A \times B .
$$

By Theorem 2, $\Sigma$ is not Zariski dense in $A \times B$. Hence, by Theorem 1 , the Zariski closure of $\Sigma$ in $A \times B$ is a proper non zero abelian subvariety $C \subset A \times B$. So $C$ is isogenous to either $A$ or $B$. In the case of assertion $3, C$ must project to zero either in $A$ or $B$, which closes the proof of this case. In the case of assertion 2 we have

$$
\Sigma \subset C \cap(A \times A)^{\sharp}=C^{\sharp} .
$$

(The equality above follows from the fact that, by Theorem 6.1 in [B2], $C^{\sharp}$ is the intersection of all $\delta$-characters $\chi$ of $C$ and, on the other hand, any such $\chi$ lifts to a $\delta$-character of $A \times A$, due to Poincaré reducibility.) Since $C$ is simple, by assertion 1 applied to $C$, we get that $\Sigma=C^{\sharp}$ and assertion 2 is proved.

The rest of the paper is devoted to the proof of Theorem 2. Assume $\Gamma$ contains a $\delta$-closed subset $\Sigma$ that is Zariski dense in $A$, with $1 \leq a(\Sigma) \leq g$ and seek a contradiction. We may assume $\Sigma$ and $\Gamma$ are irreducible. Set $G:=\Gamma^{[\infty]}$, $V:=\Sigma^{[\infty]}$. Then $G$ will be an algebraic $D$-group and $V$ will be a closed 
$D$-subvariety of $G$. Since $\Sigma$ is Zariski dense in $A$ we must have that the canonical projection $V \rightarrow A$ is dominant and, hence, generically finite. Let $W$ be the normalisation of $A$ in the field of fractions of $V$ and let $\bar{V}$ be a smooth projective compactification of $V_{\text {reg }}=($ smooth locus of $V)$ such that $D:=\bar{V} \backslash V_{\text {reg }}$ is a divisor with simple normal crossings, and the morphism $V_{\text {reg }} \rightarrow A$ extends to a morphism $\bar{V} \rightarrow A$. There is an induced morphism $p: \bar{V} \rightarrow W$. Denote by $\kappa$ the Kodaira dimension of a projective variety (by which we understand, as usual, the Kodaira dimension in the sense of $[\mathrm{I}]$, [U] of a smooth projective model of the variety.) By Theorem 13 in [Kaw], $\kappa(W) \geq 0$ and there exist an abelian subvariety $B \subset A$, etale covers $\tilde{W}, \tilde{B}$ of $W$ and $B$ respectively, and a projective normal variety $\tilde{Y}$ such that $\tilde{Y}$ is finite (and dominant) over $A / B, \tilde{W} \simeq \tilde{B} \times \tilde{Y}$, and $\kappa(\tilde{Y})=\operatorname{dim} \tilde{Y}=\kappa(W)$. Moreover, by Theorem 4 in [Kaw], if $\kappa(W)=0$ then $W$ is an abelian variety, in which case we may take, of course, $\tilde{W}=W$ and $\tilde{B}=B=A$. Due to the above discussion we are in one of the following cases:

Case 1. $\kappa(W) \geq 1$.

Case 2. $W$ is an abelian variety and $p(D)$ has codimension $\geq 2$ in $W$.

Case 3. $W$ is an abelian variety and $p(D)$ has codimension 1 in $W$.

As in [B1] we may assume there exist:

- morphisms of complex algebraic varieties $V \rightarrow A \rightarrow S, \bar{V} \rightarrow W \rightarrow A$, $\tilde{Y} \times_{S} \tilde{B} \simeq \tilde{W} \rightarrow W, \tilde{Y} \rightarrow A / B$ (where $\bar{V}$ is a smooth relative projective

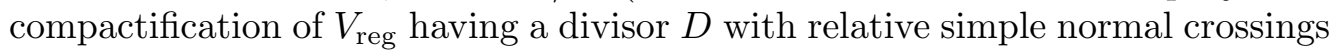
at infinity, and $A \rightarrow S$ is an abelian scheme, $B$ is an abelian subscheme, $\tilde{W}$ is etale over $W$, and $\tilde{Y}$ is finite and dominant over $A / B$.)

- a closed immersion $V \subset G$ (where $G \rightarrow S$ is a group scheme of finite type),

- nowhere vanishing vector fields $\delta$ on $S$ and $\tilde{\delta}$ on $G$, with $\tilde{\delta}$ lifting $\delta$,

having the following properties. All varieties above are flat over $S$ and $S$ is smooth. Moreover, multiplication, unit and inverse on $G$ are horizontal for $\tilde{\delta}$, the ideal sheaf of $V$ in $G$ is preserved by $\tilde{\delta}$, and there exist an extension of $\delta$-fields $(C(S), \delta) \rightarrow(\mathcal{F}, \delta)$ such that $(G, \delta), V, W, \ldots$ and the natural morphisms that we considered among them are deduced via base change from $(G, \tilde{\delta}), V, W, \ldots$ and from the corresponding morphisms.

For any closed point $s \in S$ denote by $G_{s}, V_{s}, W_{s}, \ldots$ the corresponding fibres at $s$ respectively. Let $B$ be any analytic disc in $S$ tangent to $\delta$ and let $G_{B}, V_{B}, W_{B}, \ldots$ be the corresponding analytic spaces obtained by pull back via $B \rightarrow S$. It is enough, in case 1 , to prove that, upon replacing $S$ by a Zariski open set of it, $A_{b} / B_{b}, b \in B$ fall into at most countably many isomorphism classes (for then, by Lemma 1.3 in [B1], we would get that $A / B$ descends to $\mathcal{C}$, contradicting the trace zero hypothesis.) Similarly, in cases 2 and 3 , it is enough to prove that $A_{b}, b \in B$ fall into at most countably many isomorphism classes. As in [B1], it follows, by an elementary result of Hamm about families of Lie groups, that all the fibres $V_{b}, b \in B$ are analytically isomorphic (but apriori not algebraically 
isomorphic.) In particular the smooth loci $\left(V_{b}\right)_{\mathrm{reg}}, b \in B$ are analytically isomorphic. Shrinking $S$ in the Zariski topology we may also assume that $W_{s}$ is the normalization of $A_{s}$ in the function field of $V_{s}$ and $\left(V_{\text {reg }}\right)_{s}=\left(V_{s}\right)_{\text {reg }}$ for all $s$.

Assume we are in Case 1 above. Shrinking $S$ in the Zariski topology we may assume that $\tilde{Y}_{s}$ is of general type (i.e. $\kappa\left(\tilde{Y}_{s}\right)=\operatorname{dim} \tilde{Y}_{s}$ ), and that $\tilde{W}_{s}$ is an etale cover of $W_{s}$. Let $\tilde{V}_{\text {reg }}:=V_{\text {reg }} \times_{W} \tilde{W}$. The fibres $\left(\tilde{V}_{\text {reg }}\right)_{s}$ are etale covers of $\left(V_{\text {reg }}\right)_{s}=\left(V_{s}\right)_{\text {reg }}$ hence, by the "rigidity of the fundamental group", $\left(\tilde{V}_{\text {reg }}\right)_{b}$ are analytically isomorphic for $b \in B$. Fix a point $b_{0} \in B$. Then we may consider for all $b \in B$ the non degenerate analytic maps

$$
(*) \quad\left(\tilde{V}_{\text {reg }}\right)_{b_{0}} \simeq\left(\tilde{V}_{\text {reg }}\right)_{b} \rightarrow \tilde{Y}_{b} .
$$

By Theorem 2 in $[\mathrm{KO}]$ any non degenerate analytic map from an algebraic variety into a projective variety of general type is actually algebraic. So the maps $\left(^{*}\right)$ are algebraic, hence we get algebraic dominant maps

$$
\operatorname{Alb}\left(\tilde{V}_{\text {reg }}\right)_{b_{0}} \rightarrow \operatorname{Alb} \tilde{Y}_{b} \rightarrow A_{b} / B_{b}
$$

and we are done, since $\mathrm{Alb}\left(\tilde{V}_{\text {reg }}\right)_{b_{0}}$ has only countably many quotients.

Assume we are in Case 2 above. Shrinking $S$ in the Zariski topology we may assume that $A_{s}$ is an abelian variety, and that, upon denoting by $p_{s}: \bar{V}_{s} \rightarrow W_{s}$ the natural projection, we have that $p_{s}$ is surjective and $p_{s}\left(D_{s}\right)$ has codimension $\geq 2$ in $W_{s}$ for all $s$. There is a closed subscheme $Z_{s}$ of $W_{s}$ of codimension $\geq 2$ such that $p_{s}$ induces an isomorphism $\bar{V}_{s} \backslash p_{s}^{-1}\left(Z_{s}\right) \simeq W_{s} \backslash Z_{s}$. Consider for each $b \in B$ the analytic map

$$
W_{b_{0}} \backslash\left(Z_{b_{0}} \cup p_{b_{0}}\left(D_{b_{0}}\right)\right) \simeq \bar{V}_{b_{0}} \backslash p_{b_{0}}^{-1}\left(Z_{b_{0}} \cup p_{b_{0}}\left(D_{b_{0}}\right)\right) \subset\left(V_{b_{0}}\right)_{\text {reg }} \simeq\left(V_{b}\right)_{\text {reg }} \rightarrow A_{b} .
$$

It is well known that any analytic map $f: X \backslash Y \rightarrow P$, where $X, P$ are smooth projective varieties, and $Y$ is Zariski closed of codimension $\geq 2$ in $X$, is algebraic. (Recall the argument. We may assume $P$ is a projective space. Let $H$ be a generic hyperplane in $P$. By $[\mathrm{GR}]$ p. $181, f^{*} H$ extends to a Cartier divisor $M$ on $X$. Hence the line bundle $f^{*} \mathcal{O}(1)$ extends to a line bundle $L$ on $X$. The pull backs of the sections of $\mathcal{O}(1)$ extend to sections of $L$ by the Second Riemann Theorem $[\mathrm{GR}]$ p.132. This closes the argument.) In particular we get rational maps of abelian varieties (and hence regular maps)

$$
W_{b_{0}} \rightarrow A_{b}
$$

for all $b$. So $A_{b}$ is one of the countably many quotients of $W_{b_{0}}$ and our proof in Case 2 is done.

Assume we are in Case 3. By further blowing up $\bar{V}$, we may, and will, assume in this case that the morphism $p: \bar{V} \rightarrow W$ is a succession of blowing ups with smooth centers. 
Claim 1. $V_{\text {reg }}$ is logarithmically of general type.

Here a smooth quasi projective variety $X$ is called "logarithmically of general type" if $\bar{\kappa}(X)=\operatorname{dim} X$, where $\bar{\kappa}$ denotes the logarithmic Kodaira dimension in the sense of [I]. (Recall that if $X=\bar{X} \backslash D$ where $\bar{X}$ is a smooth projective variety and $D$ is a divisor with simple normal crossings then $\bar{\kappa}(X)$ equals the " $K_{\bar{X}}+$ $D$-dimension" of $\bar{X}$.) Assume for a moment that Claim 1 is true. Shrinking $S$ in the Zariski topology we may assume that $\left(V_{s}\right)_{\text {reg }}$ is logarithmically of general type for all $s$. Propositions 2.2 and 4.2 of [Sak], together with the argument at the beginning of Section 7 in [KO], imply that any holomorphic non degenerate map from a smooth algebraic variety into a smooth quasi-projective variety which is logarithmically of general type is actually algebraic. So, in our case, we get morphisms of algebraic varieties

$$
\left(V_{b_{0}}\right)_{\mathrm{reg}} \simeq\left(V_{b}\right)_{\mathrm{reg}} \rightarrow A_{b}
$$

for all $b \in B$. Hence, again, $A_{b}$ runs through the countable set of quotients of the Albanese variety of (a smooth projective model of) $\left(V_{b_{0}}\right)_{\text {reg }}$, and we are done.

Let us check Claim 1. Let $E \subset \bar{V}$ be the (reduced) exceptional divisor of $p: \bar{V} \rightarrow W$. Let $H \subset W$ be the union of all divisorial components of $p(D)$. Claim 1 will be implied by the following

Claim 2. $H$ is ample.

Let us show that Claim 2 implies Claim 1. The canonical divisor on $\bar{V}$ has the form $K_{\bar{V}}=p^{*} K_{W}+E^{\prime}=E^{\prime}$ where $E^{\prime} \geq 0$, Supp $E^{\prime}=S u p p E$. On the other hand $p^{*} H=D+E^{\prime \prime}$ where $E^{\prime \prime} \geq 0$, Supp $E^{\prime \prime} \subset S u p p E$. There exists a positive integer $n_{0}$ such that $E^{\prime \prime} \leq n_{0} E^{\prime}$. Then for any positive $n$ we have

$$
n n_{0}\left(K_{\bar{V}}+D\right)=n n_{0} E^{\prime}+n n_{0} D \geq n E^{\prime \prime}+n D=p^{*}(n H) .
$$

Hence, if Claim 2 is true, for $n \gg 0$ the linear system $\left|n n_{0}\left(K_{\bar{V}}+D\right)\right|$ separates points outside $E \cup D$ and Claim 1 follows.

So we are left to check Claim 2. Assume $H$ is not ample. Then the identity component $W^{\prime}$ of the group of all $x \in W$ for which the translate $H+x$ is linearly equivalent to $H$ has positive dimension. Since the abelian variety $W^{\prime}$ acts on the linear system $|H|$, it acts trivially so $H+W^{\prime}=H$. Let $Z \subset W$ be a closed subset of codimension $\geq 2$ such that

$$
\bar{V} \backslash p^{-1}(Z) \simeq W \backslash Z,
$$

and let $Z^{\prime} \subset W$ be the union of all components of $p(D)$ of codimension $\geq 2$. The generic fibre of $Z \cup Z^{\prime} \rightarrow W^{\prime \prime}:=W / W^{\prime}$ has dimension at most (dim $W-$ $2)-\operatorname{dim} W^{\prime \prime}=\operatorname{dim} W^{\prime}-2$. In other words, for $x \in W$ sufficiently general, $\left(x+W^{\prime}\right) \cap\left(Z \cup Z^{\prime}\right)$ has codimension $\geq 2$ in $x+W^{\prime}$. On the other hand, since $H+W^{\prime}=H$, for $x \in W \backslash H$ the intersection $\left(x+W^{\prime}\right) \cap H$ is empty. Since 
$p(D)=Z^{\prime} \cup H$ we have, for $x \in W$ sufficiently general, that $\left(x+W^{\prime}\right) \cap(p(D) \cup Z)$ has codimension $\geq 2$ in $x+W^{\prime}$. Consider the composition

$$
\left(x+W^{\prime}\right) \backslash(p(D) \cup Z) \subset W \backslash(p(D) \cup Z) \simeq \bar{V} \backslash p^{-1}(p(D) \cup Z) \subset V \subset G .
$$

Since any rational map from a smooth algebraic variety into an algebraic group, that is defined outside a closed set of codimension $\geq 2$, must be defined everywhere, the above composition induces a regular map

$$
W^{\prime} \simeq x+W^{\prime} \rightarrow G
$$

that is generically injective. Replacing $A$ by an abelian variety isogenous to it we may assume $A=A_{1} \times \ldots \times A_{m}$, where $A_{i}$ are simple abelian varieties. Also, by enlarging $\Gamma$, we may assume $\Gamma=\Gamma_{1} \times \ldots \times \Gamma_{m}$, where $\Gamma_{i} \subset A_{i}$ are $\delta$-closed subgroups of finite absolute dimension. So $G=G_{1} \times \ldots \times G_{m}$, with $G_{i}$ algebraic $D$-subgroups of $A_{i}^{\infty}$. So there is an index $i$ such that the composition with the $i-$ th projection

$$
W^{\prime} \rightarrow G \rightarrow G_{i},
$$

is not constant. Since any regular map of an abelian variety into a commutative algebraic group is a homomorphism composed with a translation it follows that $G_{i}$ contains a non trivial abelian subvariety $B_{i}$. Since $\operatorname{Ker}\left(G_{i} \rightarrow A_{i}\right)$ is a vector group the projection $B_{i} \rightarrow A_{i}$ is injective; since $A_{i}$ is simple this projection is also surjective. So $G_{i} \rightarrow A_{i}$ has a section. Composing this section with the inclusion $G_{i} \subset A_{i}^{\infty}$ we get that the projection $A_{i}^{\infty} \rightarrow A_{i}$ has a section. By Corollary 2.5 in [B2] this implies that $A_{i}$ descends to $\mathcal{C}$, a contradiction. This proves Claim 2, hence Claim 1, hence Theorem 2.

Remarks. There is an interesting formal analogy between the model theoretic properties of differential algebraic groups and model theoretic properties of complex Lie groups. In both cases the Hrushovski-Zilber theory [HZ] leads to powerful results. Also, in both cases one may expect direct proofs of these results, free of model theory. The present paper dealt with differential algebraic groups; for the case of Lie groups we refer to [P1].

\section{Acknowledgements}

The first author is endebted to D. Marker, A. Pillay and C.Wood for extremely enjoyable and fruitful discussions on model theory, during the AMS meeting in New York, April 1996.

\section{References}

[B1] A. Buium, Intersections in jet spaces and a conjecture of S. Lang, Ann. of Math. 136 (1992), 557-567.

[B2] , Geometry of differential polynomial functions, I: algebraic groups, Amer. J. Math 115 (1993), 1385-1444.

[C] P. Cassidy, Differential algebraic groups, Amer. J. Math. 94 (1972), 891-954.

[GR] H. Grauert and R. Remmert, Coherent analytic sheaves, Springer 1984. 
[H] E. Hrushovski, The Mordell-Lang conjecture over function fields, J. Amer. Math. Soc. 9 (1996), 667-690.

[HS] E. Hrushovski and Z. Sokolovic, Minimal subsets of differentially closed fields, Trans. Amer. Math. Soc. (to appear).

[HZ] E. Hrushovski and B. Zilber, Zariski geometries, J. Amer. Math. Soc. 9 (1996), 1-56.

[I] S. Iitaka, On logarithmic Kodaira dimension of algebraic varieties, in: Complex analysis and algebraic geometry, W. L. Baily Jr and T. Shioda editors, Cambridge Univ. Press, 1977, pp. 175-191.

[Kaw] Y. Kawamata, Characterization of abelian varieties, Compositio Math. 43 (1981), 253-276.

[KO] S. Kobayashi and T. Ochiai, Meromorphic mappings into compact complex spaces of general type, Invent. Math. 31 (1975), 7-16.

[K1] E. Kolchin, Differential algebra and algebraic groups, Academic Press, New York, 1973.

[K2] _ Differential algebraic groups, Academic Press, New York, 1985.

[L] S. Lang, Division points on curves, Ann. Mat. Pura Appl. (4) LXX (1965), 229-234.

[Man] Yu. I. Manin, Algebraic curves over fields with differentiation, Izv. Akad. Nauk SSSR, Ser. Mat. 22 (1958), 737-756, Amer. Math. Soc. Transl. Ser. 2 (1964), 59-78.

[MMP] D. Marker, M. Messmer, and A. Pillay, Model theory of fields, Lecture Notes in Logic 5, Springer, 1996.

[P1] A. Pillay, Definable sets in generic complex tori, Ann. Pure Appl. Logic 77 (1996), 75-80.

[P2] _ Some foundational questions concerning differential algebraic groups, Pacific J. Math. (to appear).

[Sak] F. Sakai, Kodaira dimensions of complements of divisors, in: Complex analysis and algebraic geometry, W. L. Baily Jr and T. Shioda editors, Cambridge Univ. Press, 1977, pp. 239-258.

[U] K. Ueno, Classification of algebraic varieties and compact complex spaces, Lecture Notes in Math. 439, Springer, 1975.

Department of Mathematics and Statistics, University of New Mexico, AlbuQUERQUE, NM 87131, U.S.A.

E-mail address: buium@math.unm.edu

Department of Mathematics, University of Illinois, 1409 West Green St., Urbana, IL 61801, U.S.A.

E-mail address: pillay@math.uiuc.edu 